East African Medical Journal Vol. 82 No. 3 March 2005

NEONATAL SEPTICAEMIA IN LOW BIRTH WEIGHT INFANTS AT KENYATTA NATIONAL HOSPITAL, NAIROBI

D. E. Simiyu. MBChB, MMed (Paed) Certificate Neonatology (Tel Aviv), Lecturer. Department of Paediatrics and Child Health. Faculty of Medicine, College of Health Sciences. University of Nairobi, P.O. Box 19676. Nairobi, Kenya

\title{
NEONATAL SEPTICAEMIA IN LOW BIRTH WEIGHT INFANTS AT KENYATTA NATIONAL HOSPITAL, NAIROBI
}

\section{E. SIMIYU}

\begin{abstract}
Objective: To quantify the burden of infection among low birth weight (LBW) infants at the new born unit (NBU) of Kenyatta National Hospital (KNH), identify any risk factors, causative organisms and their antibiotic sensitivities.

Design: Retrospective study utilising case notes.

Setting: New born unit, Kenyatta National Hospital, Nairobi.

Subjects: All LBW infants admitted to the NBU of KNH from January to December 2000. Results: A total of $533 \mathrm{LBW}$ infants were admitted to the NBU. Seventy four (13.9\%) had confirmed sepsis from blood culture. The case fatality rate was $36.5 \%$. Place and mode of delivery, antenatal clinic attendance and premature rupture of membranes did not inerease the rate of sepsis. The common organisms isolated were gram negative organisms $60(66.6 \%)$, while gram positive organisms were $30(33.4 \%)$. Antibiotic sensitivity revealed high resistance to ampicillin and good sensitivity to aminoglycosides, third generation cephalosporins, clavulanic acid potentiated amoxycillin and chloramphenicol.

Conclusion: Case fatality rate for low birth weight infants with sepsis is high. Gram negative organisms are the predominant pathogens. No maternal risk factors were identified. Antibiotic sensitivity shows adequate sensitivity to aminoglycosides and third generation cephalosporins.
\end{abstract}

\section{INTRODUCTION}

Low birth weight (LBW) infants carry high morbidity and mortality from various conditions including infection. This results from either prematurity, prolonged hospital stay and increased invasive procedures such as endotracheal incubation, indwelling catheters, and increased venepunctures (I). Infectious morbidity and mortality has been shown previously to be higher in LBW infants than those of normal weight by various authors (2-5). This study aims to quantify the magnitude of infectious morbidity among LBW infants at $\mathrm{KNH}$ and identify any differences in bacteriology, mortality and predisposing factors from those reported elsewhere.

\section{MATERIALS AND METHODS}

The study was conducted at the records department of the KNH. The records of all LBW infants admitted to the NBU from January to December 2000 were retrieved. The neonatal period was defined as that period from birth upto the age of twenty eight days, while LBW was defined as birth weight less than $2,500 \mathrm{~g}$. Neonatal sepsis was defined as culture positive sepsis only.

Ethical Considerations: The study was approved by the hospital ethics and research committee.
Confidentiality was ensured by using inpatient registration numbers rather than names.

Data Management: This is a sub analysis of data collected for a general audit of morbidity and mortality of LBW infants admitted to the NBU. The data were analysed on the SPSS programme. Percentages, ratios, rates and measures of significance by Chi square and fisher exact tests were derived where appropriate.

Procedure: All neonates who had culture proven sepsis were included in the analysis. These were stratified by sex and birthweight. A data sheet was used to extract information on sex, presence of premature rupture of membranes (PROM), place of birth, mode of birth, duration of stay, maternal age, parity and marital status. The bacterial isolate, age at culture and drug sensitivity pattern were also recorded.

\section{RESULTS}

Five hundred and thirty three LBW infants were analysed. A total of $163(30.6 \%)$ blood cultures were performed of which 90 bacterial cultures were obtained. Seven had mixed growth of two organisms, while one had three organisms from a single culture. A further seven infants had culture growths at two different times during their stay in the unit. In total $74(13.9 \%)$ infants had culture proven sepsis. If the seven cultures at two 
different times are treated as separate (new infections), then the rate of sepsis was $81(15.2 \%)$. There was no positive culture of cerebral spinal fluid nor urine. A total of 27 infants with sepsis died making a case fatality rate of $36.5 \%$. There was no significant difference in the sex or birth weights between the dead and the survivors. However from the analysis, very low birth weight (VLBW) infants of weight less than $1250 \mathrm{~g}$ suffered $80-100 \%$ mortality (Table 1).

Bacteriology: The most frequently isolated bacteria were gram negative $60(66.6 \%)$ as compared to gram positive $30(33.4 \%)$. One case of group B beta haemolytic streptococci was found while there was no candida sepsis. Early onset disease (disease within five days of age) accounted for 51 (56.7\%) of the cases and for 21 (77.8\%) of the mortality. Among the gram negative bacteria, early onset disease was mainly caused by citrobacter and enterobacter while klebsiella caused both early and late disease. Among the gram positive bacteria, coagulase negative staphylococci and enterococci caused both early and late disease. Alkaligens fecalis and staplylococcus aureas caused predominantly early onset disease (Table 3 ).

Table 1

Sex, birth weight and mortality

\begin{tabular}{|c|c|c|c|c|}
\hline Variable & $\begin{array}{l}\text { Alive } \\
\text { No. (\%) }\end{array}$ & $\begin{array}{l}\text { Dead } \\
\text { No. }(\%)\end{array}$ & Chi-Square & $P$-value \\
\hline \multicolumn{5}{|l|}{ Sex } \\
\hline Male & $22(29.7)$ & $15(20.3)$ & 0.52 & 0.47 \\
\hline Female & $25(33.8)$ & $12(16.2)$ & & \\
\hline \multicolumn{5}{|l|}{ Birth weight } \\
\hline VLBW & $19(25.7)$ & $15(20.3)$ & 1.58 & 0.21 \\
\hline LBW & $28(37.8)$ & $12(16.2)$ & & \\
\hline
\end{tabular}

There was no significant difference in the rates of sepsis between infants born after PROM and those without. Similarly, antenatal clinic attendance, mode of delivery and place of delivery had no influence on the rate of sepsis (Table 2).

Table 2

Predictors of septicaemia

\begin{tabular}{|c|c|c|c|c|}
\hline Predictor & $\begin{array}{l}\text { Sepsis } \\
\text { No. }(\%)\end{array}$ & $\begin{array}{l}\text { No sepsis } \\
\text { No. }(\%)\end{array}$ & Chi-square & P-value \\
\hline \multicolumn{5}{|l|}{ PROM } \\
\hline PROM & $13(2.4)$ & $48(9)$ & 3.18 & 0.075 \\
\hline NO PROM & $61(11.4)$ & $411(77.1)$ & & \\
\hline \multicolumn{5}{|l|}{ ANC } \\
\hline ANC & $54(10.1)$ & $292(54.8)$ & 2.45 & 0.12 \\
\hline NO ANC & $20(3.8)$ & $167(31.3)$ & & \\
\hline \multicolumn{5}{|l|}{ Mode of delivery } \\
\hline SVD & $40(8.1)$ & $322(65.4)$ & 2.29 & 0.13 \\
\hline $\mathrm{CS}$ & $21(4.3)$ & $109(22.2)$ & & \\
\hline \multicolumn{5}{|l|}{ Place of delivery } \\
\hline BBA & $18(3.4)$ & $92(17.5)$ & 0.51 & 0.48 \\
\hline $\mathrm{KNH}$ & $48(9-1)$ & $304(57.7)$ & & \\
\hline Other hospital & $8(1.5)$ & $57(10.8)$ & 0.08 & 0.77 \\
\hline
\end{tabular}

PROM = Premature rupture of membranes, $A N C=$ Antenatal clinic, $S V D=$ Spontaneous vertex delivery, $C S=$ Caeserian section, $\mathrm{BBA}=$ Born before arrival 
Table 3

Bacterial profile

\begin{tabular}{llll}
\hline Bacteria & Total Isolates & $\begin{array}{l}\text { Onset }<5 \text { days } \\
\text { Early onset disease }\end{array}$ & $\begin{array}{l}\text { Onset }>5 \text { days } \\
\text { Late onset disease }\end{array}$ \\
\hline Klebsiella & 21 & 11 & 10 \\
Citrobacter & 18 & 14 & 4 \\
Enterobacter & 9 & 6 & 3 \\
Acinetobacter & 6 & 2 & 4 \\
Pseudomonas spp & 4 & 1 & 3 \\
Proteus mirabilis & 1 & 0 & 1 \\
Escherichia Coli & 1 & 0 & 1 \\
Total gram negative & $60(66.6 \%)$ & 34 & 26 \\
CONS & 17 & 9 & 8 \\
Enterococci & 5 & 3 & 2 \\
Alkaligens Fecali & 4 & 3 & 1 \\
Staph. aureus & 2 & 2 & 0 \\
Strept. Viridan & 1 & 1 & 1 \\
GBS I & 1 & 0 & 12 \\
Total gram positive & $30(33.4 \%)$ & 17 & 0 \\
\hline
\end{tabular}

CONS = Coagulase negative staphylococci, GBS= Lancefield group B beta haemolytic streptococci.

Cephalosporins (third generation) and clavulanic acid potentiated amoxycillin offered the least rates of resistance while ampicillin had the highest rate of resistance. Third generation cephalosporins, aminoglycosides, clavulanic acid potentiated amoxycillin and chroramphenicol provided the best sensitivity. Both isolates of staphylococcus aureaus were methicillin resistant (MRSA) and all except two isolates of CONS were also methicillin resistant. All staphylococci (aureus and CONS) were sensitive to vancomicin (Table 4).

Table $A$

Antibiotic sensitivity/ resistance

\begin{tabular}{lll}
\hline Antibiotic & $\begin{array}{l}\text { Sensitive } \\
\text { No. (\%) }\end{array}$ & $\begin{array}{l}\text { Resistant } \\
\text { No. (\%) }\end{array}$ \\
\hline Gentamicin & $31(34.4)$ & $8(8.9)$ \\
Clavulanate/ Amoxycillin & $34(37.8)$ & $1(1.1)$ \\
Chloramphenicol & $31(34.4)$ & $8(8.9)$ \\
Ceftazidine & $25(27.8)$ & $4(4.4)$ \\
Cefuroxime & $26(28.9)$ & $4(4.4)$ \\
Cefotaxime & $25(27.8)$ & $2(2.2)$ \\
Ampicillin & $16(17.8)$ & $19(21.1)$ \\
Methicillin & $2(2.2)$ & $10(11.1)$ \\
Lincomycin & $8(8.9)$ & $2(2.2)$ \\
Erythromycin & $3(3.3)$ & $6(6.7)$ \\
\hline
\end{tabular}

\section{DISCUSSION}

Sepsis for this study was defined strictly as culture positive. This definition means that many infants with negative culture were excluded even if clinical and other laboratory indicators were suggestive. For this definition to be effective, good laboratory back up is essential. In an earlier study in the same unit, Lusweti found that most positive cultures were obtained after 48 hours of admission (6). Many blood cultures that were carried out before this time may have been reported negative hence excluded from this study. The definition of sepsis to include clinical and other laboratory parameters such as total white blood cell count, immature to total neutrophil ratio and platelet count has been utilised before $(3,7,8)$. We could not utilise this definition because a substantial number of laboratory reports and clinical notes were missing from the files. Others have previously defined sepsis to be culture proven only $(2,9,10)$.

The rate of blood culture was $30.6 \%$ which was higher than the $13.3 \%$ reported by Mukhwana in 1997 (11) but lower than the $45.6 \%$ rate reported from neonates admitted to the general wards in the same hospital (7). The reason for the earlier reported lower rate could be because for our study, case notes were 
scanned to identify actual investigations done since relying on the presence of laboratory results in the file was not adequate as $35 \%$ of these results were missing. At the time of Mukhwana's study, there was no twenty four hour, seven days a week laboratory coverage for the wards which could have resulted in reduced rate of investigations.

There was no positive cerebrospinal fluid or urine culture found. This has also been the case previously (7). In a prospective study specifically looking at neonatal meningitis in the year 2000, Laving found only four positive cerebrospinal fluid cultures out of 84 specimens (12). The cause of this low yield in cerebrospinal fluid culture could result from the liberal use of antibiotics in the unit.

The case fatality rate for LBW infants with sepsis is high $(36.5 \%)$ compared to that found by others studying neonatal sepsis in general $(2,11,13)$. It is however lower than that found in neonates in the general wards of the same hospital (7) and that found for gram negative sepsis in the same unit. This high case fatality rate may not be a result of the sepsis itself but could result from poor or inadequate supportive care as previously suggested by Musoke and Revathi (10). Stoll in her review gives average case fatality rate for neonatal sepsis in sub-Saharan Africa as $27-56 \%$ (14). The rate found in this study is therefore well within this average. Moreover we specifically looked at LBW infants who may be more vulnerable to severe infection leading to mortality (1).

An attempt to elicit risk factors for neonatal septicaemia was not fruitfull. Premature rupture of membranes, mode of delivery, place of delivery and antenatal care attendance were not found to be significant risk factors. Lusweti while studying LBW infants to compare rates of sepsis between those born in hospital and those born before arrival also found no significant differences in the rates of sepsis between the two groups (6). Musoke and Malenga had previously drawn the same results (9).

Nathoo and co-workers in Harare-Zimbabwe reported poor antenatal care and premature rupture of membranes to be significant risk factors(15). However this Harare study did not specifically look at LBW infants but addressed neonatal septicaemia in general. LBW itself whether associated with prematurity or intra uterine growth retardation is a recognised risk factor for neonatal sepsis $(4,16)$. The mortality associated especially with very low birth weight (VLBW) sepsis is high. This was also the finding in our study where VLBW less than 1250 grammes resulted in mortality between $83 \%$ - $100 \%$. The overall rate of neonatal sepsis reported in this study is lower than that reported for LBW infants in the same unit previously (6). This could have resulted from the different definitions of sepsis. The bacteriology pattern is in agreement with that previously reported from the same unit and other studies in the developing countries in the sense, that gram negative organisms are the predominant cause $(2,6,9,17)$. There was one growth of group B - Beta haemolytic streptococci obtained. Few reports of this bacteria have been recorded from Kenyatta National Hospital and elsewhere in the developing countries $(2,12,14,16)$. While historical reviews from developed countries have demonstrated a changing pattern of bacteriology of neonatal septicaemia (14), this does not appear to be the case at KNH between 1984 and 2000 save for a diminished role of staphylococcus aureas $(9,12,17)$. This contrasts with the Harare study of 1990 , which showed staph aureus and group B beta haemolytic streptococci as the frequent isolates (15).

Early onset septicaemia was the predominant variety in the LBW infants and accounted for most of the mortality. LBW infants tend to have prolonged hospital stay and hence are predisposed to nosocomial infections including coagulase negative staphylococci (CONS) (1). This was the predominant gram positive organism. There was no case of candida sepsis reported even among the predisposed VLBW infants. This may be because it was not specifically looked for or the VLBW infants succumbed long before colonisation and later sepsis from candida (19). The predominance of klebsiella septicaemia may account for the high case fatality rate. Studies elsewhere have associated klebsiella sepsis with high mortality $(15,20)$.

The drug sensitivity is similar to that found previously in the same unit (6). It is also to an extent similar to that found for general neonatal sepsis in the same unit (10). Third generation cephalosporins, aminoglycosides (especially Gentamicin), chloramphenicol and clavulanic acid potentiated amoxycillin were the most sensitive. Ampicillin had a relatively high rate of resistance. The use of third generation cephalosporins as a first line (routine) antibiotic is however approached with caution due to the early development of resistance especially among the gram negative bacteria (10). However, despite their use in the NBU at KNH for a number of years, the sensitivity appears to have remained unchanged over a period of three to four years as shown by Musoke and Revathi in 1997 (10) and Lusweti in 1999 (6).

Study limitations: This was a retrospective study that relied on the accuracy of record keeping. This may introduce bias in the sense that where records such as laboratory reports were not filed, this will lead to under reporting of cases of sepsis. Events such as PROM if not reported may lead again to under reporting and bias the results. However, the results obtained from this study are comparable to those found from previous studies. Being retrospective, the author was not able to objectively assess maturity of the LBW infants hence the role of maturity (gestational age) was not analysed 
In conclusion the predominant organisms causing neonatal sepsis in LBW infants in the NBU - KNH are gram negative bacteria usually causing early onset disease with high mortality. The case fatality rate is higher in these LBW infants than in neonatal sepsis in general. Initial empiric treatment of LBW infants should include Gentamicin as is the present practise and a third generation cephalosporin, choramphenicol or clavulanic acid potentiated amoxycillin.

\section{ACKNOWLEDGEMENTS}

To the Director of $\mathrm{KNH}$ and the ethics and research committee of the hospital for allowing this study and its subsequent publication. Janet Musia for data entry and analysis. Susan Anyango and Fosca Osimbo for providing secretarial services.

\section{REFERENCES}

I. Nelsons Text book of paediatrics. Behrman, R. E., Kliegman, R. M.. Arvin, A. M. Editors 15th edition - Harcourt Asia PTE Ltd. 1999 Chapter 10.

2. Moreno, M. T.. Vargas, S., Poveda, R. et al Neonatal sepsis and meningitis in a developing Latin American country. Pediatr. Infect. Dis. J. 1994; 13:516-220.

3. Nehama Linder. Ohel, G., Gazit, G. et al Neonatal sepsis after prolonged premature rupture of membranes. $J$. Perinatology 1995; 15:36-38.

4. Mcintire, D. D., Bloom. S. L.. Casey. B. M. and Leveno. K. J. Birth weight in relation to morbidity and mortality among newborn infants New Eng. J. Med. 1999; 340:1234 -1238.

5. Kaushik, S. Grover. N.. Parmer, V. R. et al Neonatal morbidity in a hospital at Shimla. Indian J. Paediat. 1999; 66:15-19.

6. Lusweti. B. N. Antibiotic treatment in preterm babies born before arrival (BBA) at Kenyatta National Hospital MMed Thesis 200I, University of Nairobi.

7. Simiyu, D. E. Neonatal septicemia in the General Paediatric wards at Kenyatta National Hospital - Nairobi. Kenya Medicom (In press).
8. Seaward, P. G. R. Hannah, M. E., Myhr, T. L. et al International multicentre term PROM study: Evaluation of predictors of neonatal infection in infants born to patients with premature rupture of membranes at term. Amer. 'J. Obstet Gynecol. 1998; 179:635-639.

9. Musoke, R. N. and Malenga, G. J. Bacterial infections in neonates at the Kenyatta National Hospital nursery: A prospective study. East Afr. Med. J. 1984; 61:909-917.

10. Musoke, R. N. and Revathi, G. Emergence of multi drug resistant gram negative organisms in a neonatal unit and the therapeutic implications. J. Trop Pedir. 2000; 46:86-91.

11. Mukhwana, R. Birth survey, pattern of neonatal morbidity and mortality and audit of neonatal sepsis as seen at Kenyatta National Hospital MMed Thesis 1997, University of Nairobi.

12. Laving A. Neonatal bacterial meningitis at the new born unit of Kenyatta National Hospital. MMed Thesis 2000. University of Nairobi.

13. Adejuyigbe, E., Adeodu, O. O., Ako-Nai, K. A. et al Septicemia in high risk neonates at a teaching hospital in Ile - Ife. Nigeria. East Afr. Med. J 2001: 78:540- 543.

14. Stoll, B. J. The global impact of neonatal infections. Clin. Perinat. 1997; 24:1-21.

15. Nathoo. K. J. Mason, P. R. and Chimbria, T. H. Neonatal sepsis in a Harare hospital: aetiology and risk factors. The puerperal sepsis study group. Central Afr. J. Med. 1990; 36:150-156.

16. Gibbs, R. S. The relationship between infections and adverse pregnancy outcomes: an overview (review) Annals Perinatology. 2001; 6:153-163.

17. Kasirye - Bainda, E. and Musoke R. N. Neonatal morbidity and mortality at Kenyatta National Hospital. New born unit. East Afr. Med. J. 1992; 69:360-365.

18. Onyango. F. E., Ndinya-Achola. J. Musoke. R. N. et al. Lancefield Group B - Beta haemolytic streptococcal Infections in the newborn at Kenyatta National Hospital. East Afr. Med. J. 1984; 61:376-381.

19. Ayman, E. El - Mohammed. Lauren, J. R. el al Incidence of candida parapsilosis colonization in an intensive care nursery population and its association with invasive fungal disease. Pediat. Infect Dis. J. 1994: 13:520-524

20. Nathoo, K. J. Mason, P. R. Gwazura. I. el al Severe Klebsiella infection as a cause of mortality in neonates in Harare, Zimbabwe: evidence from postmortem blood culture. Pediat. Infect. Dis J. 1993; 12:840-844. 